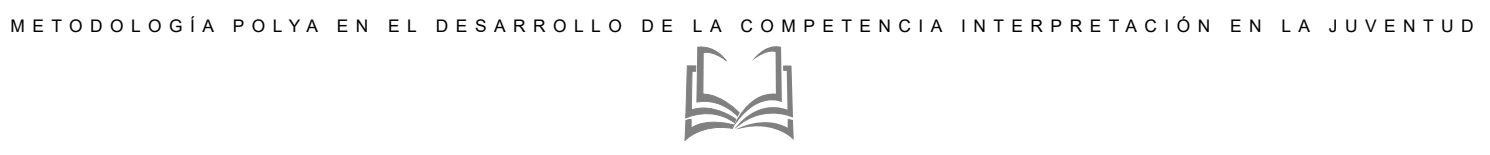

RECIBIDO EL 2 DE NOVIEMBRE DE 2019 - ACEPTADO EL 4 DE FEBRERO DE 2020

\title{
METODOLOGÍA POLYA EN EL DESARROLLO DE LA COMPETENCIA INTERPRETACIÓN EN LA JUVENTUD
}

\section{POLYA METHODOLOGY IN THE DEVELOPMENT OF COMPETENCE INTERPRETATION IN YOUTH}

\section{Jhon Jairo Meneses Patiño' \\ Mawency Vergel Ortega ${ }^{2}$ \\ Jhan Piero Rojas Suarez ${ }^{3}$}

Universidad Francisco De Paula Santander

\section{RESUMEN}

El proceso investigativo se desarrolló en la ciudad de Cúcuta, Colombia, con un grupo de estudiantes de noveno grado. El objetivo del proyecto fue diseñar e implementar una estrategia pedagógica para el desarrollo de

1 Maestrìa en Educaciòn Matemàtica, Facultad de Ciencias Bàsicas, Universidad Francisco de Paula Santander, Cúcuta, Colombia. jhonjairomp@ufps.edu.co https://orcid.org/0000-0003-1275-2165

2 Departamento de Matemáticas y Estadística, Facultad de Ciencias Básicas, Universidad Francisco de Paula Santander, Cúcuta, Colombia. mawencyvergel@ufps. edu.co

https://orcid.org/0000-0001-8285-2968

3 Facultad de Ingeniería, Universidad Francisco de Paula Santander, Colombia. 0000-0003-1275-2165, https:// orcid.org/0000-0003-2682-9880 la competencia interpretación y resolución de problemas matemáticos basada en la metodología de Polya. El método empleado para abordar el problema fue el de Solomon. El trabajo se desarrolla mediante la implementación de guías, que debieron resolverse de forma individual. Los resultados mostraron que la implementación de la metodología de Polya contribuye a la construcción de estructuras mentales que facilitan el proceso de resolución de problemas, especialmente en lo concerniente a la interpretación del problema y a la verificación de los resultados obtenidosx.

PALABRAS CLAVE: método de Solomon, resolución de problemas, interpretar, 
matemática, cálculo, competencias, estrategias enseñanza-aprendizaje.

\section{ABSTRACT}

The article is the result of a qualitative research methodology with a descriptive approach of an exploratory nature. It seeks to describe the pedagogical practices developed by teachers who implement projects of creation and innovation in the classroom and who contribute to the development of competencies in students. It was carried out at the Universidad Francisco de Paula Santander and Escuela Superior de Administración Pública in Norte de SantanderColombia, in mathematics and pre-university course's. The target population of the research is made up of students and teachers of mathematics I calculation and pre-university courses. The instrument used is a survey applied to both students and teachers of these levels. The results indicate that the pedagogical practices carried out by teachers contribute to the development of competencies in students. Compared to the competency-based approach, they consider it to be positive, because the knowledge assimilated should be used in practical life.

KEY WORDS: Solomon method, problem solving, interpret, mathematics, calculation, competencies, teaching-learning strategies.

\section{INTRODUCCIÓN}

Una de las mayores dificultades que presenta las matemáticas, es la puesta en contexto del conocimiento adquirido en el aula. Esto quiere decir que, las matemáticas son consideradas por los alumnos como un conocimiento que no es aplicable a la solución de sus problemas cotidianos. Es por ello que, se hace necesario que los maestros aborden su enseñanza de forma distinta, con diferentes modelos, mètodos, tècnicas y herramientas, de modo que, los estudiantes se interesen por su aprendizaje (Vergel, Urbina, Rojas, 2020). En este orden de ideas, abordar el trabajo del área desde la resolución de problemas puede contribuir a que dicho interés crezca y por lo tanto el aprendizaje se altamente significativo (Rojas, et al, 2019). Fundamentalmente estas dificultades se deben a la forma como se desarrolla el proceso en el aula. En sentido es posible afirmar que, a pesar de los avances de la pedagogía y la didáctica, los maestros siguen empleando metodologías tradicionales de enseñanza, que no contribuyen al mejoramiento de los procesos o se enfocan solo en el desarrollo del componente cognitivo, descuidando dimensiones presentes en su vida cotidiana fundamentales para un futuro desempeo laboral que les genere bienestar (Vergel, Rojas, Gonzàlez, 2020). En este orden de ideas, una de las principales falencias que aùn presentan los educandos, es la que tiene que ver con la resolución de problemas, puesto que esta va más allá de la simple operación de valores, lo que implica que se debe poder interpretar las soluciones obtenidas para verificar si realmente cumplen con las condiciones del problema. Como afirma Palarea, Hernández y Socas (2014).

Los alumnos utilizan como estrategia general, la tendencia a operar con los datos del problema, sin mostrar una clara comprensión del mismo y $\sin$ identificar las relaciones operacionales, conceptuales o procesuales que se dan. Aportan muchas veces soluciones que no pueden ser válidas para las condiciones del problema, lo que evidencia, además de una carencia de estrategias cognitivas (métodos heurísticos), una falta de pensamiento crítico. (p. 146). Es precisamente la superación de estos errores lo que se con la aplicación de la metodología de Polya, que va más allá de la simple operacionalización. De acuerdo con (Cruz, 2006, p. 25), Polya "Propone una serie de reglas heurísticas bastante sugerentes, pero lo más notorio consiste en que la mayoría de ellas van dirigidas a la segunda fase, de lo que él denominó su lista". Es en este sentido, que 
para resolver un problema usando el método de Polya, se deben seguir los cuatro pasos siguientes:

Entender el problema: el estudiante debe comprender el enunciado del problema, identificar las variables a trabajar, identificar si los datos son suficientes y coherentes con el problema (Martinez, Vergel y Zafra, 2016). Por esta razón en esta etapa se le formula al estudiante preguntas como: ¿entendió el problema? ¿Cuáles son los datos del problema? ¿Puede replantear el problema con sus propias palabras? ¿los datos son suficientes para comenzar a resolver el problema? (Cárdenas y Gonzales, 2016, p. 47). Es decir, se debe hacer una interpretación del enunciado, con la finalidad que elementos presenta el problema y como estos se interrelacionan. En la segunda etapa configuración de un plan, "el estudiante debe pensar en la estrategia más adecuada para resolver el problema planteado, por ello se le presentan una serie de estrategias para que de acuerdo con el problema el escoja la más adecuada" (Cárdenas y Gonzales, 2016, p. 47). Esto implica, el problema no se resuelve de forma casual, sino siguiendo una estrategia bien concebida.

En seguida se deberá resolver el modelo planteado, para culminar con la verificación de la solución encontrada. "En esta etapa el estudiante debe comprobar su respuesta, por medio de las operaciones matemáticas realizadas para hallar su respuesta y justificar la misma. En la plataforma se realizan preguntas como: ¿Es posible encontrar una solución diferente? ¿Puede encontrar otra estrategia para resolver el mismo problema? (Cárdenas y Gonzáles, 2016, p. 48). La última fase es crítica, porque de nada sirve encontrar una serie de valores si a estos no se les da sentido, pero sobre todo si no se hace una verificación de que realmente es la solución buscada y que esta cumple con los requerimientos del problema (Martinez, Vergel y Pilonieta, 2019). En conclusión, Polya establece una estrategia bien definida para abordar $y$ desarrollar un problema, lo que en teoría, debe contribuir a la estructuración de los procesos mentales.

\section{METODOLOGÍA}

El proceso investigativo se desarrolló mediante la aplicación del enfoque cuantitativo que en palabras de Monje (2011) està "orientada por conceptos empíricos medibles, derivados de los conceptos teóricos con los que se construyen las hipótesis conceptuales (Nieto, Martinez y Vergel, 2016). El análisis de la información recolectada tiene por fin determinar el grado de significación de las relaciones previstas entre las variables (Vergel et al, 2019). El diseño es de tipo cuasi-experimental, en el que se "examinan relaciones de causa y efecto entre las variables independiente y dependiente" (Sousa, Driessnack, y Costa, 2007, p. 4), pero sin manipularlas, es decir, se busca relacionarlas sin modificarlas de forma intencional.

Con el objeto de superar, una de las dificultades que presenta el diseño cuasi-experimental, el cual es el sesgo de los datos obtenidos (Rojas, Vergel, Largo, 2020), se propuso el uso del método de los cuatro grupos de Solomon (Vergel et al, 2019). Para el desarrollo de este método se asignó el total de los sujetos del estudio a los cuatro grupos, en forma aleatoria; en el primer grupo se hace una medición "antes" de la variable dependiente, luego se aplica el tratamiento experimental y finalmente se hace una medición "después", en el segundo grupo se hacen mediciones "antes" y "después" sin aplicar el tratamiento experimental; el tercer grupo recibe el tratamiento experimental y se hace en él una medición "después"; y, finalmente, en el cuarto grupo se hace solo una medición "después". (Briones, 1998, p. 11). Una vez conformados los cuatro grupos y realizado el proceso de intervención y evaluación, se 
establecen las relaciones entre la información recolectada, proceso que es complejo desde el punto de vista estadístico y que se resume en el gráfico 1.

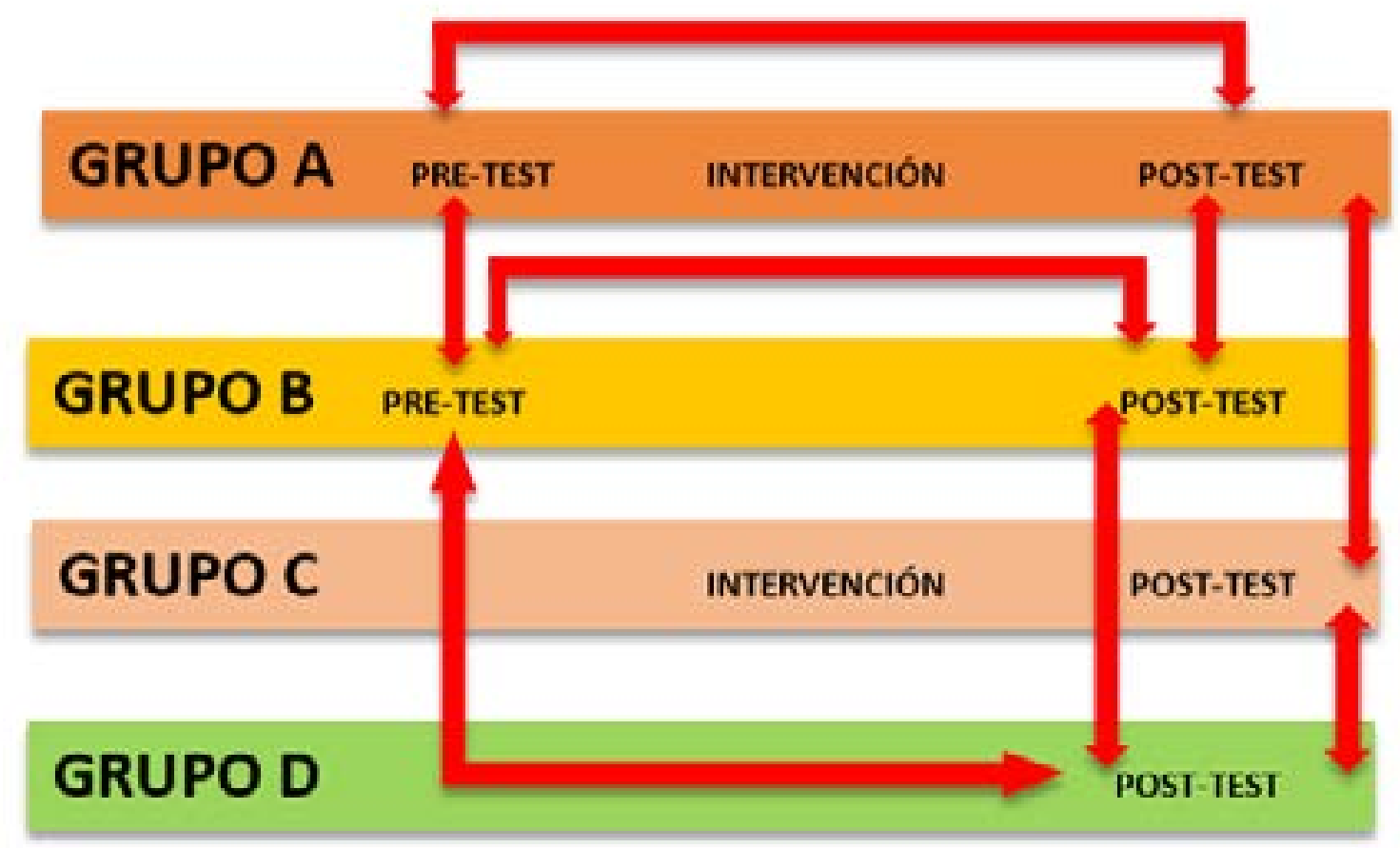

Figura 1. Análisis de la información método de Solomon. Fuente: autores

La poblaciòn objeto de estudio, la constituyen jòvenes con edades entre 13 y 16 años de edad. La muestra aleatoria, la comprenden 120 jòvenes con edades entre 13 y 16 años de edad, que cursan grado noveno en instituciones de la ciudad de Cùcuta- Colombia. La investigación se llevó a cabo en tres fases, en la primera fase se realizó un pre-test, su objetivo fue realizar un diagnóstico sobre el nivel en que se encontraban los alumnos en la competencia interpretativa en la resolución de problemas. En la segunda, se diseñó e implementó una estrategia pedagógica en buscó fortalecer la resolución de problemas en contexto. Por último, en la fase tres, se aplicó un post-test encaminado a medir la efectividad de la propuesta implementada, específicamente en la competencia resolución de problemas.

\section{RESULTADOS}

El aplicar el pre-test a dos grupos permitiò comprender si los resultados encontrados en ellos son similares o tienen un alto grado de diferenciación, de manera que se pudiese o no afirmar que los dos inician o no en el mismo nivel. En este sentido se encontró que la media de un grupo A fue de 10,31 y la del grupo B 10,52. Además, las desviaciones estándar fueron 0,488 y 0,733 respectivamente. Esto implica que los dos grupos empiezan en condiciones similares, como lo demuestra el factor ANOVA, cuyo valor $p$-valor fue 0,223 , mayor que 0,05 , lo que indica que se permite considerar que las dos medias son similares, como se muestra figura 2. 

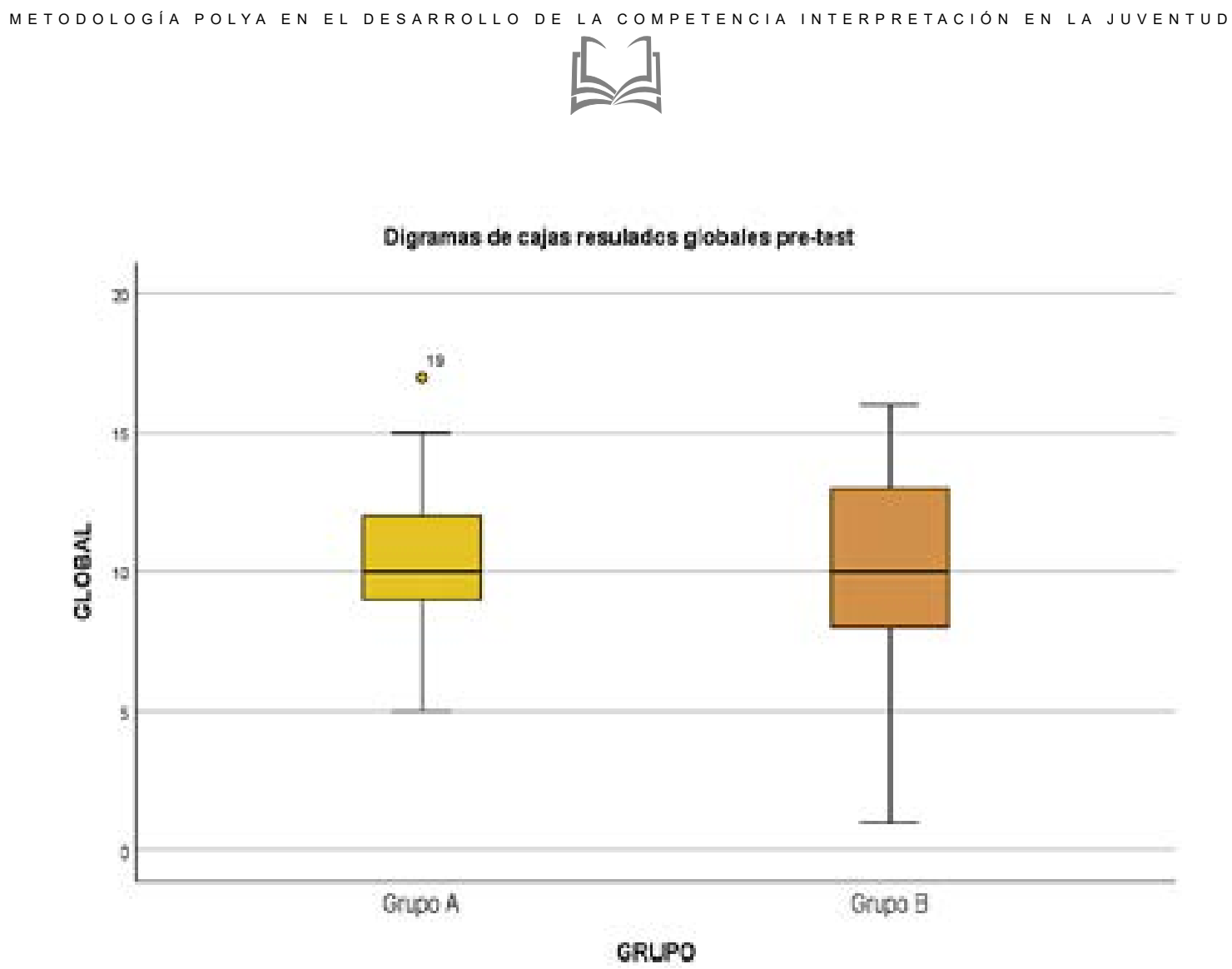

Figura 2. Comparación pre-test grupos A y B. Fuente: autores

De otra parte, resultados de la prueba final, evaluados los cuatro grupos permiten observar que la media para el grupo A fue 11,71 , mientras que el grupo B obtuvo 10 puntos en promedio. De igual forma el grupo $C$ alcanzó un promedio de 9,32 y el D 12,68. Esto implica que el grupo D, quienes sòlo presentaron post-test fue quien mejor media obtuvo, seguido del grupo A que realizó el proceso completo. De acuerdo con esto el mejoramiento fue pequeño. Sin embargo, es posible afirmar que, existe mejora, puesto que en el aspecto que más se avanzó el grupo A (media 3,98) fue en la interpretación del problema, competencia objeto de la investigaciòn. Asimismo, la mayor fortaleza del grupo D fue la ejecución del plan (media 4,07), lo que implica que el maestro se concentra en las operaciones y no en la interpretación. Esto significa que los conocimientos adquiridos por el grupo D no fueron significativos, puesto que, al no desarrollarse el proceso de interpretación, se presentaràn dificultades cuando tengan que enfrentarse a nuevas situaciones problema.
Al revisar el grupo C, grupo a quienes se realizò intervención y post-test, la media más alta por proceso se centrò en el entendimiento del problema (media 3,09), mientras que, en el grupo $B$, que presentó pre y post-test los dos procesos más fuertes fueron el entendimiento del problema y la concepción del plan, lo que es una referencia clara a la incidencia del pretest en la solución del post-test. En definitiva, se puede afirmar que la metodología de Polya contribuyó en el mejoramiento de un aspecto fundamental en el desarrollo de problemas matemáticos asociados al entendimiento del problema.

\section{CONCLUSIONES}

Después de analizados los resultados del pre-test se puede concluir que el nivel de competencia de los educandos en la resolución de problemas presenta un nivel bajo en cada uno de los dos grupos de estudio, esto implica que es un proceso que no ha sido debidamente desarrollado dentro del aula y por lo tanto 
se requiere de una intervención que busque mejorarlo, puesto que la resolución de problemas es el puente entre el conocimiento matemático y su aplicación al contexto.

Al igual que en los resultados globales, en cada uno de los pasos propuestos por Polya, los resultados encontrados son bajos, encontrando la mayor debilidad en el entendimiento del problema que es fundamental, al igual que en la concepción del plan y la visión retrospectiva de los resultados. En cuanto a la ejecución del plan, se pude afirmar que los valores encontrados no son óptimos y que difieren levemente de los otros, lo que muestra que el trabajo de aula se direcciona a la ejecución de operaciones, es decir que no se estimula el nivel de razonamiento de las situaciones problema.

Por último, se puede afirmar que al iniciar los dos grupos en un nivel de competencia similar, permitirá a futuro establecer si el proceso de intervención que se diseñó e implementò dio los resultados esperados, de igual forma, al contar en el post-test con cuatro grupos se verifica el nivel de incidencia que tiene la presentación del pre-test, así como el de la estrategia pedagógica.

En cuanto al análisis del post-test, se pudo verificar que los mejores resultados lo obtuvieron el grupo D quienes solo presentaron la prueba final, pero se evidenció que la fortaleza del grupo estuvo centrada en la ejecución del plan, esto permite inferir que este es el aspecto que más se trabaja en el aula. De otraparte, el grupo $A$, que realizo los tres procesos, obtuvo mejores resultados que el $B$, que presentó las dos pruebas, pero no fue intervenido, lo que significa que la estrategia contribuyo a la mejora. Por último, la comparación entre el grupo A y el $C$, intervenidos y presentaron el post-test, obtuvieron mejores resultados, esto implica que la presentación del pre-test, influyó en los resultados finales.
La implementaciòn de la metodologìa Polya, mejora la interpretaciòn en los jòvenes de edades entre 13 y 16 años al incluir pretest al inicio del proceso educativo.

\section{REFERENCIAS BIBLIOGRÁFICAS}

Briones, A. G. (1998). Investigaciones o diseños experimentales. La sociología en sus escenarios. Volumen 2, pp-pp 1-18. Recuperado de: https://tinyurl.coml yenarryv

Cárdenas, D. C. y González, G.D. (2016). Estrategia para la resolución de problemas matemáticos desde los postulados de Polya mediada por las TIC, en estudiantes del grado octavo del instituto Francisco José de Caldas. Tesis de maestría, Universidad Libre de Colombia, seccional Bogotá.

Cruz, A.M.J., Coronel, R. C. y Guevara, G.J. D. (2016). Comprensión lectora en resolución de problemas matemáticos en alumnos universitarios.

De Zubiría, J. (2006). Las competencias argumentativas. La visión desde la educación. Bogotá: Cooperativa Editorial Magisterio.

Hurtado, J. (2010). Metodología de la investigación. Guía para la comprensión Holística de la Ciencia. (Cuarta edición). Caracas: Ediciones Quirón.

Rojas-Suarez J P et al (2019). J. Phys.: Conf. Ser. 1414012006

Rojas-Suarez J.P., Vergel, M. Largo, F. (2020). La empatía en el aprendizaje de los estudiantes de educación básica desde la perspectiva de la interacción social. Bogotà: ECOE Ediciones. 
Martinez, J., Vergel M, Zafra S. (2016). Comportamiento juvenil y competencias pro-sociales Bogotá: Editorial Ibañez.

Martinez, J., Vergel M, Pilonieta L (2020). Lineamientos de enseñanza a estudiantes con hipoacusia. Bogotá: Ecoe Ediciones.

Monje, C. (2011). Metodología de la investigación cuantitativa y cualitativa. Neiva: Universidad Sur colombiana, 113.

Nieto, J.F. Martínez-Lozano, J.J., Vergel-Ortega, M. (2016). Validez de instrumento para medir el aprendizaje creativo. Revista Comunicaciones en estadística. 9(2), 239-254.

Ortega, A, Vergel, M. y Martinez, J. (2018). Validity of microrubri, instrument to measure the development of competences in mathematics.

Socas, M., Hernández, J., y Palarea, M. M. (2014). Dificultades en la resolución de problemas de atemáticas de estudiantes para profesor de educación primaria y secundaria.

Sousa, V., Driessnack, M., y Costa, I. (2007). Revisión de diseños de investigación resaltantes para enfermería. Parte 1: Diseños de investigación cuantitativa. Rev Latino-am Enfermagem, 15(3), 1-6.

Vergel-Ortega $M$ et al (2019). Cathedral bell's San José de Cúcuta: Heritage and acoustics. J. Phys.: Conf. Ser. 1329012004

Vergel-Ortega, M. Rojas-Suárez J. P., y González-Mendoza, J. A. (2020) Modelo estructural de correlación entre prácticas saludables y estrategia, en instituciones de salud de la frontera colombo- venezolana», Aibi revista investig. adm. ing., pp. 69-75

Vergel Ortega, M., Urbina, y Rojas, J.P. (2020). Imaginarios de educación superior en estdiantes sordos. Bogota: Ecoe Ediciones 